\title{
A Spectral Resolution study in Femtosecond Stimulated Raman Scattering Microscopy
}

\author{
Rajeev Ranjan ${ }^{1,2 *}$, Maria Antonietta Ferrara $^{2}$, and Luigi Sirleto ${ }^{1}$ \\ ${ }^{1}$ National Research Council (CNR), Institute of Applied Sciences and Intelligent Systems, Napoli, Italy \\ ${ }^{2}$ CHT @Erzelli, Nanoscopy Istituto Italiano Di Tecnologia, Genova, Italy
}

\begin{abstract}
The simultaneous mapping and the specificity of different chemical species are desirable in several biological and biomedical applications. The stimulated Raman Scattering technique is a proven and wellestablished label-free method to map the distributions of individual species in a multi-component-based system due to the linear dependence of signals on concentration and its chemical selectivity. In this framework, spectral resolution, i.e., the ability to distinguish closely lying resonances, plays a fundamental role. Here in this work, the cross-correlation of Ti:Sa \& OPO femtosecond laser beams in a stimulated Raman scattering microscope is measured. The separation between protein and lipid bands in the $\mathrm{C}-\mathrm{H}$ region is important for biochemical research and is successfully classified.
\end{abstract}

\section{Introduction}

Over the past ten years, stimulated Raman scattering (SRS) has been investigated in nanophotonic [1-4] as well as in biophotonics as an analytical, label-free, noninvasive technique with unique cellular and tissue imaging capabilities [5-8]. Stimulated Raman Scattering (SRS) based spectroscopy is a quite popular and proven technique for the spectrochemical analysis of molecules. When the SRS technique is combined with microscopy, it brings a mass revolution in tiny molecules imaging and differentiation between specific molecules. It offers high chemical selectivity of unlabelled living cells and realtime 3-D imaging with high spatial resolution and sensitivity [5-8].

The C-H stretching (2800-3100 $\left.\mathrm{cm}^{-1}\right)$ region of Raman spectra of biomolecules is the most used in SRS microscopy. Lipids and proteins can be investigated in this region, taking advantage of $\mathrm{CH}_{2}$, near $2845 \mathrm{~cm}^{-1}$, and $\mathrm{CH}_{3}$, near $2930 \mathrm{~cm}^{-1}$, Raman bands, respectively. The difference between the peaks of these two Raman bands and their width spectral shapes is about $100 \mathrm{~cm}^{-1}$. This means that they are partially overlapped. Typically, SRS microscopy is implemented by using two Fourier transform-limited (FTL) tunable picosecond $(p s)$ laser sources, which have a high spectral resolution (about 10 $\mathrm{cm}^{-1}$ ). Still, the drawback is that $p s$ pulses show a low peak intensity, thus needs high laser power for imaging [5-8]. When $f_{s}$ (femtosecond) laser sources are used, an improvement of about one order of magnitude of the SRS signal has been demonstrated with respect to ps pulses [9]. Still, a low spectral selectivity is obtained, and multi-band excitation can occur, not allowing, in principle, the separation of some bands of particular interest in biologies, such as those of lipids and proteins. To solve this issue, a number of methods for SRS multicolor imaging, based on broadband femtosecond pulses, have been developed [10-14].

In order to enhance spectral resolution in SRS microscopy based on fs laser pulses, an option is given by spectral focusing (SF). The equally chirping pump and Stokes beams with an energy spacing corresponding to the Raman line (IFD, $\Omega=\omega \mathrm{p}-\omega \mathrm{s}$ ) spectrally focuses the excitation energy into a single resonance. We note that the bandwidth $\delta \Omega$, in the limiting case, can simulate the $p s$ SRS system. Nevertheless, due to a large number of parameters, the resulting spectral resolution of SF-SRS setups is often worse than theoretically predicted [14].

It is worth noting that in coherent Raman scattering, there is a trade-off between the best spectral resolution and the best ratio of image contrast and signal intensity, which is obtained when the spectral resolution and the width of the Raman lines under observation are almost the same [15]. This condition is satisfied in the fingerprint region in the case of excitation with both ps pulses, since they match the Raman linewidths $\left(5-20 \mathrm{~cm}^{-1}\right)$, while in the case of the $\mathrm{C}-\mathrm{H}$ region, broader bandwidth $f_{s}$ pulses can be considered as excitation for lipids and proteins Raman bands.

It is well known that, due to the effects of chromatic dispersion and nonlinearities, a pulse can gain a chirp, for example, through propagation in a transparent medium. Therefore, the optical elements embedded in an SRS optical circuitry microscope setup can also change the pulse width. In this paper, taking advantage of the small chirping introduced by simply propagating the beams through dispersive materials already present in the SRS microscope setup, we demonstrate that we can be distinguished protein and lipid bands in the $\mathrm{C}-\mathrm{H}$ region.

\footnotetext{
${ }^{*}$ Corresponding author: rajeev.ranjan@iit.it
} 


\section{Experimental Set-up}

In our previous paper, we have successfully described the design and execution of femtosecond-based SRS microscopy, which is able to cover the $\mathrm{C}-\mathrm{H}$ region $\left(>2,500 \mathrm{~cm}^{-1}\right)$ [16-22]. In Figure 1, a femtosecond-SRS spectroscopy setup is integrated with a Nikon C2 confocal microscope, which comprises an inverted Nikon Tieclipse microscope and a scan head. This arrangement has been described in full in our earlier publications. A Ti:Sapphire (Ti:Sa-Chameleon Ultra II-pulse duration $=140 \mathrm{fs}$, repetition rate $=80 \mathrm{MHz}, \lambda=680-1080 \mathrm{~nm}$ ) and a femtosecond optical parametric oscillator (OPOChameleon Compact OPO-pulse duration $=200 \mathrm{fs}$, repetition rate $=80 \mathrm{MHz}, \lambda=1000-1600 \mathrm{~nm}$ ) are used in this configuration.

Our SRS microscope allows us to probe individual Raman bands at a time. By tuning the wavelength of the pump or Stokes beam with the matching Raman frequency of different samples at the required Raman band, we can obtain the images of the different Raman band matching molecules with high chemical contrast [16-23]. Thus, $\mathrm{CH}_{2}$ $\left(2,845 \mathrm{~cm}^{-1}\right)$ and $\mathrm{CH}_{3}\left(2,940 \mathrm{~cm}^{-1}\right)$ stretching signals can be collected at one Raman shift at a time, leading in principle to map the distributions of the lipid and protein contents in the same image. We note that when $f s$ pulses are used, the loss in spectral selectivity of SRS signal is not prejudicial in lipids imaging, so SRS images acquired at $2,845 \mathrm{~cm}^{-1}$ may be mainly attributed to the lipid. On the contrary, the chemical specificity of SRS images at 2,940 $\mathrm{cm}^{-1}$ is not particularly efficient. Since both lipids and proteins have significant Raman signals and their Raman bands are partially overlapped, SRS images acquired at $2,940 \mathrm{~cm}^{-1}$ contain both lipids and protein signals.

Considering that SRS is a two-pulse technique, its spectral resolution is not defined by the spectrum of the individual exciting pulses but by the spectrum of their temporal interference. Consequently, cross-correlation is fundamental for providing information about the entire system; in particular, the FWHM of pump and probe beams' cross-correlation allows us to evaluate the experimental spectral bandwidth [23].

Taking advantage of a non-linear process, when both pulses spatially and temporally overlapped, a crosscorrelation pulse signal can be generated. Since the rate of two-photon absorption (TPA) is also proportional to the product of two intensity laser beams with different wavelengths, this process is suitable for cross-correlation measurements. Taking advantage of TPA, crosscorrelation measuring is carried out, focusing two different beams into a detector. The average photocurrent is larger when the two beams are temporally aligned than when they are misaligned. The detected photocurrent usually has a non-zero background level because the two beams can produce TPA separately even when nonoverlapped. In this phenomenon, the output signal is equal to the cross-correlation of the input signals.

Cross-correlators based on TPA has some significant advantages: (i) a higher sensitivity can be obtained with respect to second harmonic generation ( $\mathrm{SHG})$, due to the involvement of fields only at the original frequency, $\omega$, owing to the TPA resonant second-order transition nature; (ii) extremely short pulses can be characterized since TPA can operate in a wide wavelength range not restricted by the narrow phase-matching bandwidth; (iii) in TPA nonlinear signal multiplication and detection are combined into one process, leading to a simplification and a higher efficiency with respect to a two-step process of optical nonlinearity followed by linear detection.

A pulseCheck 50 (APE, Berlin) is used in our measurements, with a pulse width measurement range [10 fs-12 ps]. The pulse Check connects to the PC via USB, thanks to APE's standardized software interface, which can be remote-controlled or integrated into automated setups. An extra flip-flop mirror in our existing optical architecture is introduced (see figure 1) to establish crosscorrelation measurements. The flip-flop mirror (FFP/AM) is installed in-between the mirror M4 and M5 so that each laser beam can be directly diverted into an autocorrelator. Once laser beams are directed into the autocorrelator, the beam is tuned to stabilize and maximize the intensity. After reaching the maximum intensity, the pulse is recorded, and the data analysis is performed. For the data analysis, we used the Gaussian fitting curve function cftool of MATLAB 2020.

For this measurement, a step-by-step acquisition method is utilized. Probe and pump beams are focused inside the TPA detector. The Ti:Sa is optically delayed by an optical delay line (Newport MOD MILS200CC) inserted between the Ti:Sa and the microscope. The delay line moves one step and stops, and when it stops during the process, the signal is acquired. The single or averaged signal decay is available in recorded files.

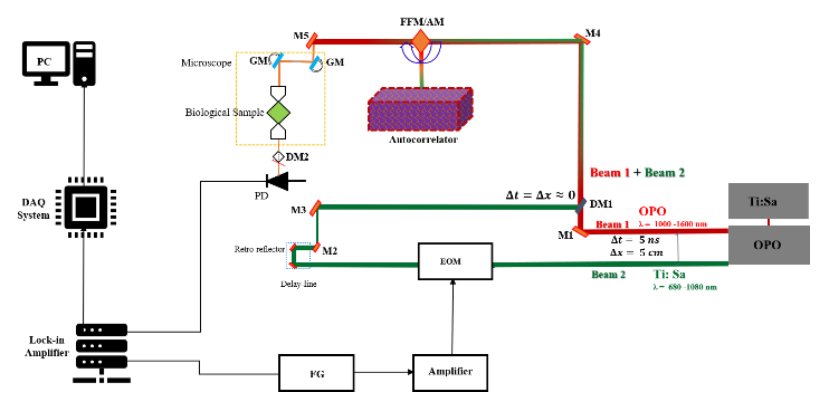

Fig. 1. Optical architecture for spectral characterization single beam

\section{Results and Discussion}

The measured FWHM (Full width at half maximum) of our Ti:Sa and OPO cross-correlation was $262 f_{S}$ (see Figure 2) [22]. Thus, the obtained experimental spectral bandwidth, given by the FWHM of cross-correlation in the frequency domain, was $56 \mathrm{~cm}^{-1}$. 


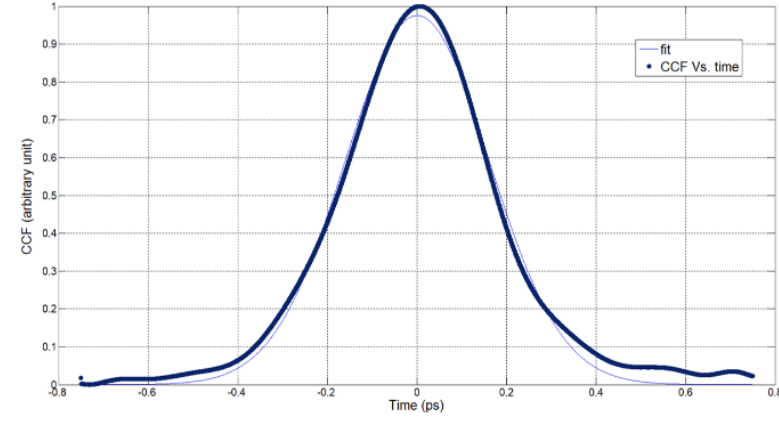

Fig. 2 Cross-correlation of Ti:Sa-OPO and its Gaussian fit

Since our experimental spectral bandwidth was $56 \mathrm{~cm}^{-1}$, when we set the $\lambda_{\text {Ti:Sa }}$ at $808 \mathrm{~nm}$ and $\lambda_{\mathrm{OPO}}$ at 1049 to excite the $2845 \mathrm{~cm}^{-1}$ lipid band, but in-fact we are exciting from (FWHM) 2817 to $2873 \mathrm{~cm}^{-1}$; in the same way, when we set the lasers beams of $\lambda_{\text {Ti:Sa }}$ at 803 and $\lambda_{\text {OPO }}$ at 1052 to excite the protein band at $2940 \mathrm{~cm}^{-1}$, then effectively we exciting the range 2912-2968 $\mathrm{cm}^{-1}$ (FWHM). In Figure 3, spectral bandwidth deriving by the chirping of the pulses (56 $\mathrm{cm}^{-1}$ - continuous lines) and two simulated Raman bands having the two peaks at a distance of $95 \mathrm{~cm}^{-1}$ with the width of about $100 \mathrm{~cm}^{-1}$ (in figure 3 blue and red) are illustrated. The overlap between two excited bandwidths is highlighted with yellowish shadow; however, they contribute to the Raman signal from this region can be neglected considering that the intensities are lower than FWHM values and, so, they can be regarded as under threshold. Thus, we can conclude that using $56 \mathrm{~cm}^{-1}$ chirped pulses, the $2845 \mathrm{~cm}^{-1}$ channel is essentially related to the lipid signal, and the $2940 \mathrm{~cm}^{-1}$ channel is principally due to protein content. Based on this explanation, we may conclude that our SRS microscope is suited for molecular imaging specificities such as lipids, proteins, and fatty acids in the $\mathrm{C}-\mathrm{H}$ region.

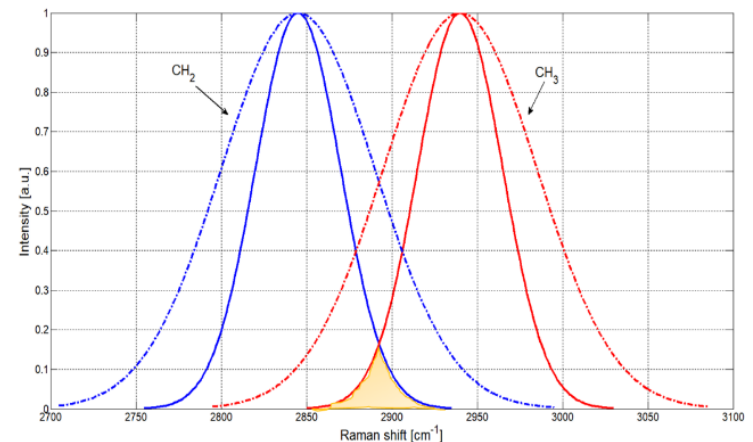

Fig. 3. Raman bands for $\mathrm{CH}_{2}\left(2845 \mathrm{~cm}^{-1}\right.$, red circles $)$ and $\mathrm{CH}_{3}$ (2940 $\mathrm{cm}^{-1}$, blue diamonds).

In SRS microscopy, the trade-off between high selectivity offered by picoseconds pulsed laser source and high SRS signal obtained by using femtoseconds pulses is still an open question, and it is widely investigated. In particular, there is interest in label free image both the lipids and proteins distribution for biological application. Unfortunately, the Raman bands of these two components are very close. Thus, ps pulses are required; however, the protein content could be low, leading to very weak Raman signals so that fs pulses would be appropriate.

In this paper, an alternative method is proposed to overcome the drawback of spectral focusing. Its basic idea is to avoid adding optical elements in the SRS microscopy optical setup and taking advantage of chirping, introduced by simply propagating the beams through dispersive materials already present in the SRS microscope. The pros are that no additional optical elements have to be introduced in the experimental setup, giving the significant advantages of a more cheap and straightforward design. The cons are that the spectral resolution is fixed. However, in our set up an experimental value of $56 \mathrm{~cm}^{-1}$ for spectral resolution is measured by cross-correlation techniques, and molecular specificity is demonstrated for lipids and proteins in the $\mathrm{C}-\mathrm{H}$ region.

\section{Acknowledgements}

The authors would like to thank M. Indolfi and V. Tufano (ISASI-CNR) for their technical support.

\section{References}

1. Sirleto, L.; Ferrara, M.A.; Nikitin, T.; Novikov, S.; Khriachtchev, L. Giant Raman gain in silicon nanocrystals. Nat. Commun. 2012, 3, 1-6. doi: 10.1038/ncomms2188;

2. Sirleto, L.; Vergara, A.; Ferrara, M.A. Advances in stimulated Raman scattering in nanostructures. Adv. Opt. Photon. 2017, 9, 169-217. doi: 10.1364/AOP.9.000169

3. M.A. Ferrara, L.Sirleto, G.Nicotra, C.Spinella, I.Rendina. Enhanced gain coefficient in Raman amplifier based on silicon nanocomposites. 2011 vol.9, pp.1-7. doi:10.1016/j.photonics.2010.07.007.

4. Sirleto, L.; Aronne, A.; Gioffrè, M.; Fanelli, E.; Righini, G.C.; Pernice, P.; Vergara, A. Compositional and thermal treatment effects on Raman gain and bandwidth in nanostructured silicabased glasses. Opt. Mater. 2013, 36, 408-413. http://dx.doi.org/10.1016/j.optmat.2013.10.001

5. Freudiger, C.W.; Min,W.; Saar, B.G.; Lu, S.; Holtom, G.R.; He, C.; Tsai, J.C.; Kang, J.X.; Xie, X.S. Label-Free Biomedical Imaging with High Sensitivity by Stimulated Raman Scattering Microscopy. Science 2008, 322, 1857-1861. [CrossRef] [PubMed]

6. 6. Cheng, J.-X.; Xie, X.S. Vibrational spectroscopic imaging of living systems: An emerging platform for biology and medicine. Science 2015, 350, aaa8870. [CrossRef]

7. Camp, C.H., Jr.; Cicerone, M.T. Chemically sensitive bioimaging with coherent Raman scattering. Nat. Photon 2015, 9, 295-305. [CrossRef] 
8. Zumbusch, A.; Langbein,W.; Borri, P. Nonlinear vibrational microscopy applied to lipid biology. Prog. Lipid Res. 2013, 52, 615-632. [CrossRef]

9. Zhang, D.; Slipchenko, M. N.; Cheng, J.-X. Highly sensitive vibrational imaging by femtosecond pulse stimulated Raman loss. J. Phys. Chem. Lett. 2011, 2, 1248-1253.

10. Fu, D.; Lu, F.K.; Zhang, X.; Freudiger, C.W.; Pernik, D.R.; Holtrom, G.; Xie, X.S. Quantitative chemical imaging with mul-tiplex stimulated Raman scattering microscopy. J. Am. Chem. Soc. 2012, 134, 3623-3626. [CrossRef]

11. Liao, C.-S.; Wang, P.; Wang, P.; Li, J.; Lee, H.J.; Eakins, G.; Cheng, J.-X. Spectrometer-free vibrational imaging by retrieving stimulated Raman signal from highly scattered photons. Sci. Adv. 2015, 1, e1500738. [CrossRef]

12. Fu, D.; Holtom, G.; Freudiger, C.; Zhang, X.; Xie, X.S. Hyperspectral Imaging with Stimulated Raman Scattering by Chirped Femtosecond Lasers. J. Phys. Chem. B 2013, 117, 4634-4640. [CrossRef]

13. Pegoraro, A.F.; Ridsdale, A.; Moffatt, D.J.; Jia, Y.W.; Pezacki, J.P.; Stolow, A. Optimally chirped multimodal CARS micros-copy based on a single Ti:sapphire oscillator. Opt. Express 2009, 17, 29842996. [CrossRef]

14. Rocha-Mendoza, I.; Langbein,W.; Borri, P. Coherent anti-Stokes Raman microspectroscopy using spectral focusing with glassdispersion. Appl. Phys. Lett. 2008, 93, 201103. [CrossRef]

15. Brückner, L.; Buckup, T.; Motzkus, M. Exploring the potential of tailored spectral focusing. J. Opt. Soc. Am. B 2016, 33, 1482-1491.[CrossRef]

16. D'Arco A., Brancati N., Ferrara M. A., Indolfi M., Frucci M., and Sirleto L., Biomed. Opt. Express, 2016, 7, (5), pp. 1853-1864, (2016).

17. Annalisa D'Arco, Maria Antonietta Ferrara, Maurizio Indolfi, Vitaliano Tufano and Luigi Sirleto., Journal of Nonlinear Optical Physics \& Materials Vol. 26, No. 4, 1750052 ((2017).

18. R. Ranjan, A. D'arco, M. A. Ferrara, M. Indolfi, M. Larobina and L. Sirleto. Opt. Exp., Vol. 26, Issue 20, pp. 26317-26326, (2018).

19. Ranjan, R., Indolfi, M., Ferrara, M. A., Sirleto, L. J. Vis. Exp. (149), e59614, (2019).

20. Ferrara MA, Filograna A, Ranjan R, Corda D, Valente C, Sirleto L. PLOS ONE 14(5): e0216811, (2019).

21. R. Ranjan, M. A. Ferrara, A. Filograna, C. Valente and L. Sirleto. Journal of Instrumentation, vol. 14, P09008, (2019).

22. Sirleto, L.; Ranjan, R.; Ferrara, M.A. Appl. Sci., 11, 3903, (2021).
23. T.; Obara, Y.; Misawa, K. Single-beam phasemodulated stimulated Raman scattering microscopy with spectrally focused detection. J. Opt. Soc. Am. B 2017, 34, 1004. [CrossRef] 